\title{
Current State of Affairs with Regard to Continuing Medical Education for Technicians in Radiology in Francophone West Africa
}

\author{
Abdoulatif Amadou1 ${ }^{*}$, Massaga Dagbe2, Sonia Bignon Mahussi Gwladys Adjadohoun², \\ Pihou Gbande ${ }^{4}$, Lantam Sonhaye ${ }^{1}$, Lama Kedigoma Agoda-Koussema ${ }^{5}$, Komlanvi E. Adjenou ${ }^{1}$
}

\author{
${ }^{1}$ Radiology Department of University Hospital Center of Campus, Lomé, Togo \\ ${ }^{2}$ Radiology Department of University Hospital Center of de Kara, Kara, Togo \\ ${ }^{3}$ Radiology Department of University Hospital Center of Cotonou, Cotonou, Benin \\ ${ }^{4}$ Radiology Department of Regional Hospital Center of Sokode, Sokode, Togo \\ ${ }^{5}$ Radiology Department of University Hospital Center of Sylvanus Olympio, Lomé, Togo \\ Email: *amadoulatif@yahoo.fr
}

How to cite this paper: Amadou, A., Dagbe, M., Adjadohoun, S.B.M.G., Gbande, P., Sonhaye, L., Agoda-Koussema, L.K. and Adjenou, K.E. (2021) Current State of Affairs with Regard to Continuing Medical Education for Technicians in Radiology in Francophone West Africa. Open Journal of Radiology, 11, 91-100.

https://doi.org/10.4236/ojrad.2021.113009

Received: April 6, 2021

Accepted: August 28, 2021

Published: August 31, 2021

Copyright $\odot 2021$ by author(s) and Scientific Research Publishing Inc. This work is licensed under the Creative Commons Attribution International License (CC BY 4.0).

http://creativecommons.org/licenses/by/4.0/

(c) (i) Open Access

\begin{abstract}
Background: This study aimed to determine the level of continuing medical education (CME) of medical imaging technicians in French-speaking West Africa. Materials and methods: This opinion survey was administered to radiology technicians in French-speaking West Africa from $1^{\text {st }}$ June to $31^{\text {st }}$ October 2020, i.e. over a period of 5 months concerning their level of CME and their need for CME. Results: Out of 100 technicians, $69 \%$ were men. The mean age was 40 years, with extremes ranging from age 23 to age 57 . The majority of technicians (64\%) worked in the public sector and $78 \%$ had work experience of less than 11 years. Most technicians were holders of a Bachelor's degree (73\%). The types of CMEs they knew were congresses, seminars, and modular training in $46 \%$ of the cases. The most available CMEs were seminars (63\%). All technicians agreed on the need for CME and 96\% said it had an impact on the delivery of radiology services. Technicians who had received CME at least once represented $61 \%$. For $90 \%$ of technicians, the specific CME sought was management in radiology (33.33\%) and radiation protection (25.81\%). Conclusion: The need for CME is increasingly indisputable to radiology technicians in French-speaking West Africa; however, it remains a luxury for them, due to its scarcity and its high cost.
\end{abstract}

\section{Keywords}

Continuing Medical Education, Technicians, Radiology, French-Speaking West Africa 


\section{Introduction}

Continuing professional development is a learning process that helps professionals in various fields to acquire new skills during their working life with the aim of returning to or maintaining employment and in order to secure or optimize career paths [1]. It is the most important way to adapt the professional behavior of health workers to current needs or to take into account current trends. This is all the more true in medical imaging where technological advances are permanent, disrupting the techniques and protocols in this field [2]. The medical imaging technician must adapt by relying on solid fundamental knowledge, from which he must constantly adapt his practice, develop and broaden his skills [3]. A study in New Zealand found that $84 \%$ to $93 \%$ of technicians agreed to acquire new knowledge in medical imaging [4]. In Africa, few studies have been devoted to CMEs of radiology and medical imaging technicians. A study in Togo on the state of play of continuing education for radiology technicians reported that $90.38 \%$ of technicians found initial training insufficient and all technicians affirmed the need for continuing education with a preference for training leading to a diploma [5]. Based on this observation, it seemed necessary to us to extend this study to several African countries with the general objective of determining the level of CME of medical imaging technicians in French-speaking West Africa.

\section{Materials and Methods}

\subsection{Type and Period of Study}

Our survey was conducted in 6 French-speaking West African countries (Benin, Burkina-Faso, Côte d'Ivoire, Niger, Senegal, and Togo). This was an opinion survey carried out from $1^{\text {st }}$ June to $31^{\text {st }}$ October 2020, over a period of 5 months.

\subsection{Study Site}

The study took place in the medical imaging department of French-speaking West Africa.

\subsection{Methods}

We included in the study radiology and medical imaging technicians working in French-speaking West Africa. We designed the questionnaire in two formats, namely a physical copy and a digital copy via Google Forms, in order to collect the opinions of radiology technicians in French-speaking West Africa on the current state of affairs with regard to their continuing education. We first assessed the validity of the questionnaires before submission to the technicians.

\subsection{Main Variables}

The questionnaire requested for the following information: general characteristics (age, gender, country of practice, sector of activity, professional practice) and specific characteristics (technician training, advice on continuing training 
and its impact on care of the patient, participation in continuing training, accessibility to training, financing of training, job prospects).

\subsection{Data Collection}

We selected the participants by referring to the database of the association of radiology technicians of French-speaking Sub-Saharan Africa. The sample size was defined as $50 \%$ of the participants, with a margin of error of $10 \%$.

We considered that the level of medical training was high when the technician had participated in more than 5 training sessions, medium when the technician had participated in between 2 and 5 training sessions, low when the technician had participated in less than 2 training sessions.

\subsection{Statistical Analysis}

The results were recorded and presented using Excel and Word software from the 2016 office pack and processed by Epi Info version 7.2 software. Bibliographic references were generated with Zotero software. The anonymity and confidentiality of information have been respected.

\section{Results}

\subsection{General Characteristics}

A total of 100 technicians were included in the study. The mean age was 40 , with extremes ranging from age 23 to age 57. Males represented $69 \%(n=69)$ with a male/female ratio of 2.22:1. Technicians with less than 11 years of professional experience constituted $78 \%$.

\subsection{Country of Practice}

Technicians working in Togo represented 36\% (Figure 1).

\subsection{Business Sector}

Technicians in the public sector represented $64 \%$, as compared to $29 \%$ for the private sector (Figure 2).

\subsection{Opinion on CME}

Technicians estimated that CME was necessary in $57 \%$ of cases $(n=57)$ and absolutely essential in $43 \%$ of cases $(n=43)$.

\subsection{Knowledge about Types of CME}

The types of CME that technicians knew about were congresses, seminars and modular training in $46 \%$ of cases (Figure 3).

\subsection{Types of CME Available}

Seminars were the most readily available CME according to $63 \%(n=63)$ of technicians (Figure 4). 


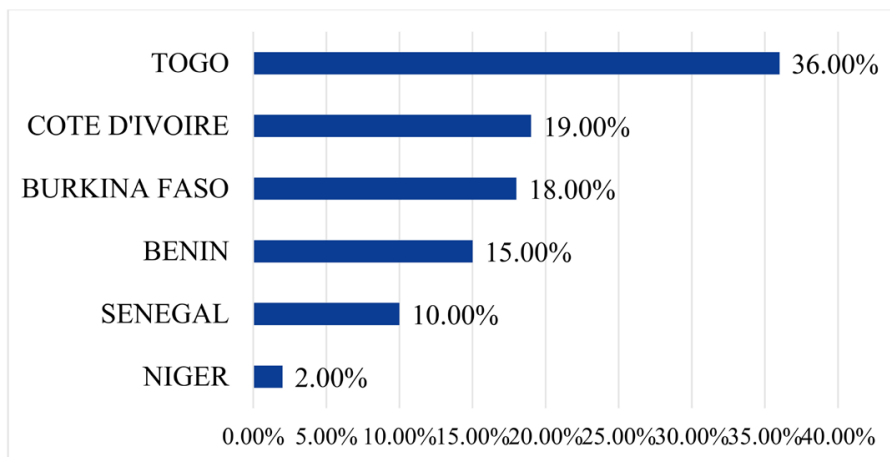

Figure 1. Distribution of technicians by country of practice.

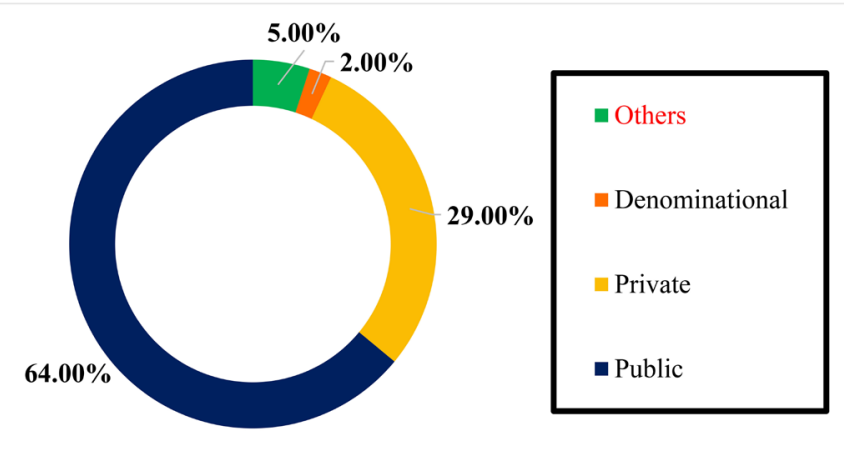

Figure 2. Distribution of technicians by business sector.

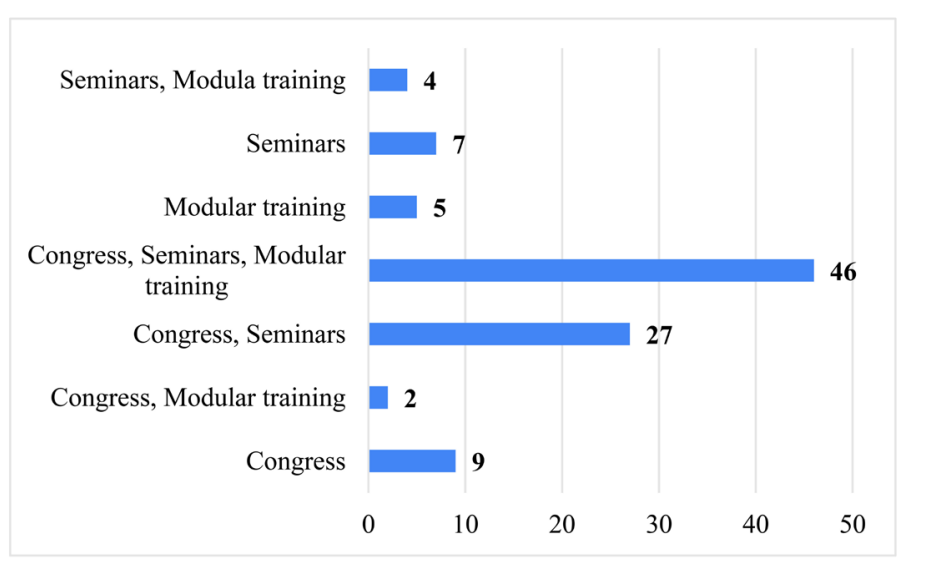

Figure 3. Distribution of technicians according to knowledge of types of CME.

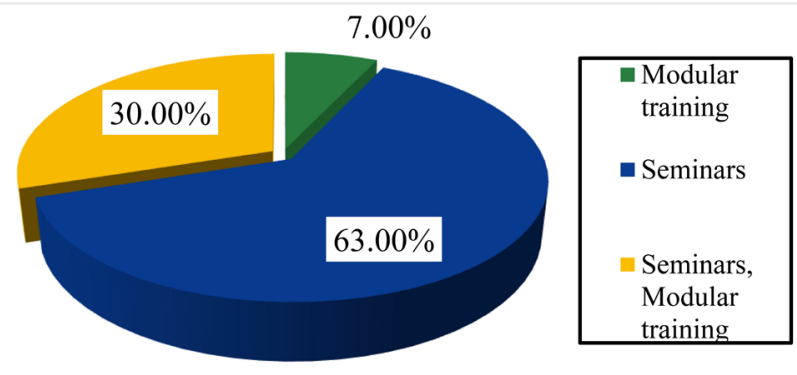

Figure 4. Type of CME available. 


\subsection{Need to Do CME after Initial Training}

Among the technicians, $48 \%(\mathrm{n}=48)$ felt it was necessary to do CME after the initial training while $52 \%(n=52)$ believed there was no need for it.

\subsection{Participation in a CME}

$61 \%(n=61)$ of technicians had received continuing education after graduation. Among the technicians, $9 \%$ had a high level of continuing education, $26 \%$ had a medium level and 65\% had a low level.

The types of CME they received were dominated by congresses (26\%) (Figure 5).

Their goal for participating in CME was to improve their skills for 55.22\% (n $=34$ ) of them (Figure 6).

\subsection{Funding of Continuing Education}

Funding of continuing education was totally the responsibility of $24 \%(\mathrm{n}=24)$ of technicians, fully funded by the institution in $11 \%(n=11)$ of cases and partially funded by the institution for $26 \%(n=26)$ of technicians.

\subsection{Accessibility to Continuing Education}

Access to continuing education was difficult for $84 \%(n=84)$ of technicians.

Among technicians, 90\% $(\mathrm{n}=90)$ felt that CME was necessary to specialize. Among them, 33.33\% would like to benefit from management training in radiology and medical imaging, and $25.81 \%$ would like training in radiation protection (Figure 7).

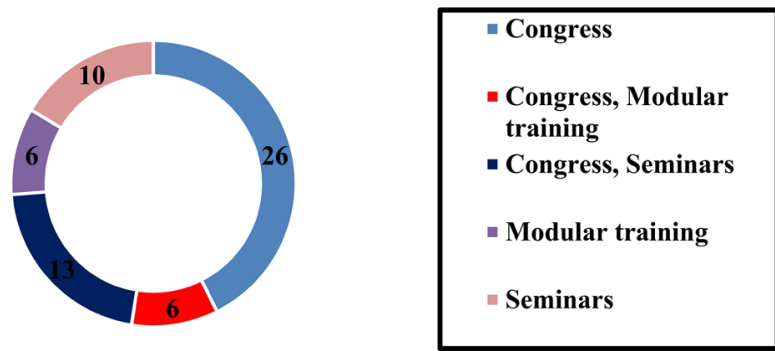

Figure 5. Type of continuing training followed by technicians.

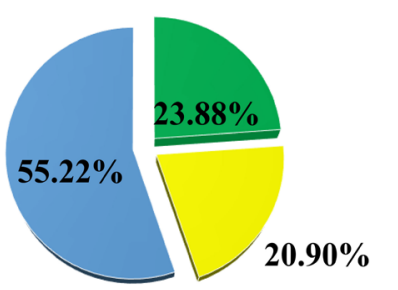

- Acquisition of new qualifications

Adaptation to new Technologies

- Skill improvement

Figure 6. Distribution of technicians according to motivation for continuing education. 


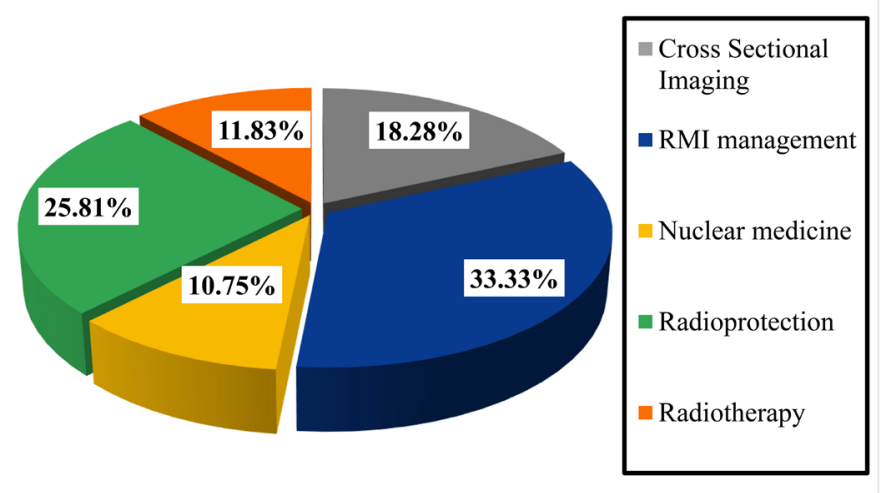

Figure 7. Distribution of technicians according to the type of continuing education desired.

\section{Discussion}

Our study was an opinion survey on the current state of affairs of continuing medical education (CME) for technicians from 7 countries in French-speaking West Africa. We collected the opinions of 100 technicians, $64 \%$ of whom worked in the public sector. We used two channels (electronic and physical) to reach our target population. These two routes are perfectly compatible with the research protocols in medicine, allowing us to achieve the objectives of our study.

Nevertheless, we noted two limitations. The first relates to the very small number of participants in some countries, which prevents us from generalising the results obtained. The second limitation has to do with the electronic method used, which not only could mislead some employees when filling in the questionnaire, but also did not allow us to have access to employees who do not have electronic devices (computer, tablets, smartphones).

The uniqueness of our study lies in the fact that it is one of the very first to be carried out in French-speaking West Africa on the state of play of continuing education for technicians in radiology and medical imaging.

The mean age of technicians was 40 years and there was a predominance of men (male/female ratio of 2.22:1). In the study by Yielder et al. [4], $85.3 \%$ of technicians were between 30 and 59 years old, with a predominance of women (83.2\%) and the majority working in public radiology departments (67\%). The predominance of the public sector could be explained by the fact that it is the largest employer of health care personnel including radiology technicians in Africa as well as in developed countries.

\subsection{Participation in Continuing Education}

All the technicians in our study had recognized the importance of CME to better practice the profession of radiology and medical imaging technician. Thus for radiology technicians, $\mathrm{CME}$ is an essential factor of development and progress in order to maintain a standard of competency necessary to adapt to the demands of the profession. $61 \%$ of technicians had already participated in a CME, reflect- 
ing their desire for continuing education, which is noteworthy since radiology is a constantly evolving field. In the study by Yielder et al. [4], 27\% of technicians had advanced knowledge in radiology, of which the majority (88\%) had received CME in New Zealand and $12 \%$ in the United Kingdom, and $81 \%$ of them had a background in ultrasound.

Despite the willingness of most technicians to participate in a CME, it was observed that access to CME remains difficult according to $84 \%(n=84)$ of them. This is was due to the lack of financial means and the non-availability of CME. In our study, continuing education was fully funded by $24 \%$ of technicians themselves, and partially funded by $26 \%$ of technicians. These results could be explained by the absence of a scheme set up by institutions to care for the CME of technicians. This forces technicians to go for training at their own expense. The study by Yielder et al. [4] also highlighted the lack of financial resources as an impediment on CME for technicians.

\subsection{Continuing Education and Job Prospects}

\section{Need for Specific Continuing Education}

During our study, $90 \%$ of technicians expressed the need to undergo continuing training. Thus, it is obvious that continuing training is necessary to equip technicians with skills needed to cope with the constant evolution of technology, computer science, as well as the computerization and digitization of radiological equipment. In our study, management in radiology and medical imaging together with training in radiation protection were the most desired fields for CME. According to Bengana [6], concerning the administrative field, the "Diplôme universitaire de management en imagerie médicale libérale" (University diploma in management of private practice medical imaging) will enable the technician to integrate or be an administrative head of an imaging department. According to him, the new role of the medical imaging technician will take several forms. These include: acquisition of specific medical training in ultrasound in order to offset the deficit in patient contact time, specialization in cross-sectional image processing for vascular reconstructions or virtual navigation, acting as a network manager who would be in charge of the maintenance of the RIS (radiological information system) and the PACS (picture archiving and communication system), would manage access authorizations and would be the contact person for IT suppliers. Other responsibilities include management of the risk of exposure of personnel and patients to radiation under the direction of a radiation protection officer (RPO), considering that the constraints in terms of X-ray monitoring are becoming increasingly burdensome. According to Dillenseger [7], the specializations to which technicians aspire are numerous and varied, including ultrasonography, dosimetry, interventional radiology, quality control in electro-radiology, network management in electro-radiology, research associate in electro-radiology, etc. There are genuine needs that have to be met in all these fields. However, until 2015, specialization in electro-radiology was available online only. Indeed, in the absence of academic and statutory recognition, spe- 
cialization does not exist.

\section{Conclusion}

Continuing education has become more than a necessity and an obligation for health care workers in general and for radiology and medical imaging technicians in particular in the face of increasing progress in technology. All technicians agree on the importance of CME. Unfortunately, the lack of financial resources and the non-availability of most types of CME make continuing medical education inaccessible.

\section{Conflicts of Interest}

The authors declare no conflicts of interest regarding the publication of this paper.

\section{References}

[1] Abbatt, F.R. and Mejía, A. (1990) La formation continue des personnels de santé. OMS, Genève, p. 189.

[2] Grenier, N. (2013) Une évolution et une révolution dans la profession de manipulateur. Health Management, 6, 1.

[3] Prentakis, A.G., Stefanoyiannis, A.P., Georgiadis, K., Coleman, L., Foley, S.J., Herlig, D., Kollas, P., Kowalik, A., Tomczak, J. and Chatziioannou, S.N. (2016) Education, Training, and Professional Issues of Radiographers in Six European Countries: A Comparative Review. JEur CME, 5, Article ID: 31092.

[4] Yielder, J., Young, A., Park, S. and Coleman, K. (2014) Establishing Advanced Practice for Medical Imaging. New Zealand J Med Radiat Sci, 61, 14-21.

[5] Touglo, Y. (2017) La formation continue des techniciens en radiologie au Togo. Mémoire de Master, Université de Lomé, Lomé, 55 p.

[6] Bengana, A. (2013) L'Evolution Du Rôle Du Manipulateur Health Management. Health Management, 6, 1.

[7] Dillenseger, J.P. (2016) Être manipulateur d'électroradiologie médicale à l'horizon 2025. SOINS Cadres, 25, 43-46. https://doi.org/10.1016/j.scad.2015.12.010 


\section{Survey Form}

Please, we are entreating you to complete a questionnaire which will take you just about ten minutes and which is of course completely anonymous. The data collected will be used exclusively for this study.

There are no right or wrong answers to the following questions; only your opinion matters to us in order to better evaluate the answers.

Thank you for your participation.

Instruction: Tick the corresponding answer box then fill in the spaces reserved for open questions.

1) Age.....................

2) Sex

Male

Female

3) Certificate

TSRIM

IIRIM

4) Nationality

5) In what year did you complete your training?.

6) In which institution do you work?

Public

Private

Denominational

Others (indicate)

7) Did you start practicing right at the end of your training?

8) Do you think that your initial training is sufficient to practice your profession?

Yes

No

9) What do you think of continuing education?

Absolutely essential

Necessary

Not necessary

10) What are the different types of continuing education that you know?

\section{Congress}

Seminars

Modular training

Other (indicate)

11) Do you think that it is necessary to do continuing education after the initial training?

Yes

No 
12) In your opinion, does continuing education have an impact on the delivery of RMI (radiology and medical imaging) services?

Yes

No

13) In your opinion, does continuing education contribute to improvement in patient care?

Yes

No

14) Have you done any since you graduated?

Yes

No

15) If yes, what type of continuing education?

Congress

Seminar

Modular training

Other (indicate)

16) If yes, which country?

Togo

Africa (indicate the country)

Europe (indicate the country)

Other (indicate)

17) If yes, how did you finance this continuing education?

Financed by myself

Fully financed by institution

Partially financed by institution

Other (indicate)

18) Is it easy to have access to continuing education?

Yes

No

19) If no, why?

Non-availability of continuing education in this country

Lack of financial resources

Employer's refusal

Others (indicate)

20) Is there a budget for continuing education in your institution?

Yes

No

21) If yes, what is the contribution of your health facility to continuing education? 